\title{
Osteosarcomatous differentiation in lung metastases from a malignant phyllodes tumour of the breast
}

\author{
H Tsubochi, N Sato, M Kaimori, T Imai
}

J Clin Pathol 2004;57:432-434. doi: 10.1136/icp.2003.012336

Osteosarcomatous differentiation of a phyllodes tumour in the breast is extremely rare. A 54 year old woman presented with a painless lump in her left breast and a mastectomy was performed. Microscopically, the tumour was diagnosed as a malignant phyllodes tumour, where no osteosarcomatous component was found. One year later, two lung tumours were revealed upon chest $x$ ray and were surgically treated. The tumours revealed osteosarcomatous features, and were thought to be metastases from the phyllodes tumour. The present case is very unusual in that osteosarcomatous features are present only in the metastatic lesion of the phyllodes tumour.

O steosarcomatous differentiation of a malignant phyllodes tumour is rare, accounting for $1.3 \%$ of phyllodes tumours in the breast. ${ }^{1}$ The histological features of a metastatic lesion of a malignant phyllodes tumour are generally similar to those of the primary tumour. $^{12}$ We report a malignant phyllodes tumour with an unusual aspect: only lung metastases revealed osteosarcomatous differentiation, which was not found in the primary breast tumour.

"Osteosarcomatous differentiation of a malignant phyllodes tumour is rare, accounting for $1.3 \%$ of phyllodes tumours in the breast"

\section{CASE REPORT}

A 54 year old postmenopausal woman was admitted because of a painless lump in her left breast. She had no past history of malignancy and no history of prior irradiation to the breast and chest region. Physical examination indicated a movable mass, $8 \mathrm{~cm}$ in diameter, in the upper half of the left breast. The axillary lymph nodes were not palpable. Mammography indicated no calcification and no other radiological signs of malignancy. Magnetic resonance imaging demonstrated non-homogeneous signal intensity with a hypointense area and internal septation in T2 weighted images; in dynamic magnetic resonance imaging, the mass was gradually and markedly enhanced. There was no evidence of metastasis to the lungs, bones, or brain. Laboratory findings were all within the normal ranges including tumour markers (CA15-3, carcinoembryonic antigen). Aspiration breast cytology was carried out, but a diagnosis was not made. A subcutaneous mastectomy was performed and adjuvant chemotherapy or radiotherapy were not administered after the surgery.

One year after mastectomy, chest $x$ ray and a computed tomography (CT) scan demonstrated bilateral pulmonary tumours with calcification; the left mass in the upper lobe measured $3 \mathrm{~cm}$ and the right mass in the middle lobe was $2.5 \mathrm{~cm}$. The patient complained of no symptoms. Local recurrence was not found upon physical examination. A bone scintigram, brain CT, and abdominal CT were unremarkable. A one stage operation for bilateral lung tumours was performed under thoracoscopy; partial resection of the right lung and segmentectomy of the left upper division were performed. No adjuvant treatments were administered after lung resection. The patient is alive and disease free two years after surgery to the lungs.

\section{PATHOLOGY}

The surgical specimen of the breast tumour was $9 \times 7 \times 6 \mathrm{~cm}$ in size. The tumour displayed extensive infiltration into the extramammary adipose tissue, but was macroscopically free from all surgical margins. Microscopically, stromal cellularity was moderate in most areas of the tumour and stromal overgrowth was observed (fig 1). The stromal component revealed fibrosarcomatous features comprising atypical spindle cells. Atypical cells having atypical nuclei with prominent nucleoli and mitotic figures were seen (mitotic count, 15/10 high power fields) (fig 1). Foci of necrosis and myxoma-like areas were also found. An epithelial component was seen only in the peripheral area of the tumour and revealed mild hyperplasia. Immunohistochemically, the tumour cells were positive for vimentin but negative for cytokeratin, S-100, CD34, and HER2. Immunohistochemistry for p53 can be useful to distinguish malignant phyllodes tumour from benign tumours because, in contrast to benign tumours, increased p53 immunoreactivity is frequently present in the stromal cells of malignant phyllodes tumours. ${ }^{3}$ In our present case, moderate to strong p53 nuclear staining was seen in about half of the stromal cells. Based on the morphological and histological examination, the breast tumour was diagnosed as a malignant phyllodes tumour. No osteosarcomatous component or bone formation was found in the tumour, although the tumour specimen was examined macroscopically and microscopically in detail.

The left and right lung tumours were stony hard and measured $3.5 \times 3 \times 3 \mathrm{~cm}$ and $2.5 \times 2 \times 2 \mathrm{~cm}$, respectively. The tumour displayed extensive infiltration into the lung tissue. Necrosis was not observed. Histology of both the lung tumours revealed osteosarcomatous features, and an epithelial component was not seen. Trabeculae of woven bone surrounded by proliferation of atypical osteoblasts were present (fig 2). Foci of malignant appearing cartilage were also noted. Based on the histological findings, the pulmonary tumours were diagnosed as extraskeletal osteosarcoma and were thought to be metastases from the phyllodes tumour. The immunohistochemical findings for the lung tumours

Abbreviations: $\mathrm{CT}$, computed tomography 


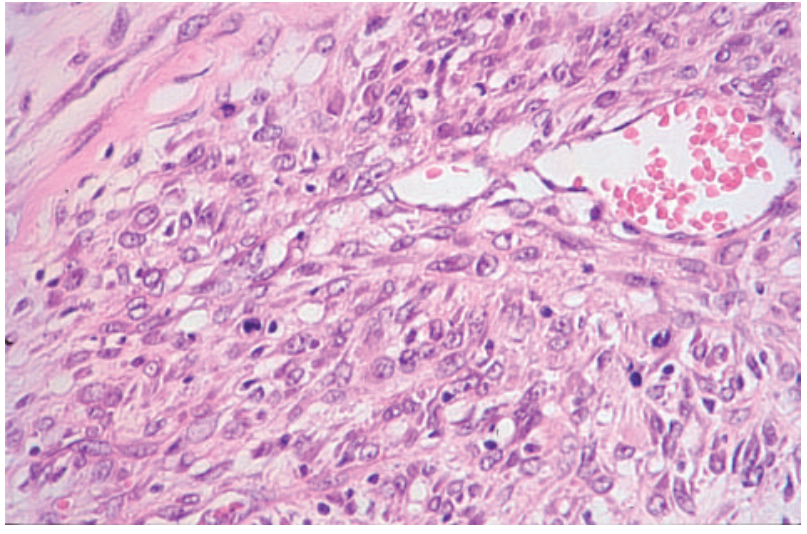

Figure 1 Microscopic findings of the breast tumour (haematoxylin and eosin stain). Hypercellular stromal overgrowth, nuclear pleomorphism, and mitoses are present.

were similar to those of the primary breast tumour with regard to vimentin, cytokeratin, S-100, CD34, and HER2. However, p53 nuclear staining, which was positive in the primary breast tumour, was not detected in the metastases in the lungs.

\section{DISCUSSION}

In approximately $22 \%$ of cases, malignant phyllodes tumours give rise to haematogenous metastases. ${ }^{4}$ The most frequent sites of metastases are the lungs, soft tissue, bone, and pleura. $^{25}$ In most cases, the metastases resembles the sarcomatous component of the primary tumour. ${ }^{12}$ Our present case is very unusual in that only the metastatic lesions in the lungs revealed osteosarcomatous features, and there was no osteosarcomatous component in the primary tumour. One report described a local recurrence of a phyllodes tumour presenting with widespread differentiation into a teleangiectatic osteosarcoma, which was not apparent in the primary tumour. ${ }^{6}$ In that case, osteosarcomatous local recurrence occurred after the patient had undergone local but complete excision of a phyllodes tumour twice in the past. However, to our knowledge there are no reports that show osteosarcomatous differentiation only in a metastatic lesion of a phyllodes tumour. The reason why only the lung metastases revealed osteosarcomatous features is unclear; however, it is possible that there were undetectable small

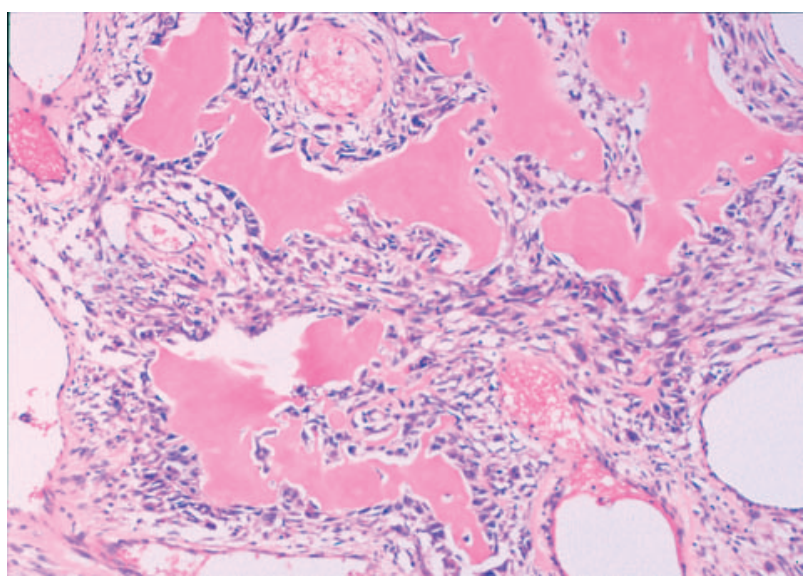

Figure 2 Microscopic findings of the lung tumour (haematoxylin and eosin stain). Metaplastic bone formation and proliferation of atypical osteoblasts are seen.

\section{Take home messages}

- To our knowledge, this is the first report of a phyllodes tumour with osteosarcomatous differentiation only in the metastatic lesions

- Careful follow up of this patient is necessary because the histological findings of both the primary and metastatic lesions include some factors associated with an unfavourable prognosis

lesions differentiating into osteosarcoma in the primary breast tumour and that they had a high metastatic potential.

Primary pulmonary osteosarcoma is extremely rare; to date, only 13 cases have been reported in the literature. ${ }^{7}$ Therefore, two primary osteosarcomas are not likely to occur simultaneously in the lungs. In addition, there was no evidence of other malignancies that might metastasise to the lungs. Thus, these two lung tumours were thought to be metastases from the malignant phyllodes tumour in the breast. It may be difficult to demonstrate directly by clonal studies that the lung tumours originate from the phyllodes tumour of the breast, because the genotype of the lung tumours may differ from that of the primary phyllodes tumour. Indeed, p53 immunoreactivity was seen only in the primary lesion and was absent from the lung tumours.

"It is possible that there were undetectable small lesions differentiating into osteosarcoma in the primary breast tumour and that they had a high metastatic potential ${ }^{\prime \prime}$

Previous studies have attempted to define those histological features that would be useful in predicting the metastatic potential of a phyllodes tumour. ${ }^{1258}$ Hawkins et al showed that four features-high mitotic count, stromal overgrowth, severe nuclear pleomorphism, and infiltrating marginswere useful predictors for the development of metastases. ${ }^{2}$ They also showed that the most reliable predictor was the presence of stromal overgrowth. According to them, patients whose primary tumours contained areas of stromal overgrowth had a $72 \%$ risk of metastatic spread, whereas those without stromal overgrowth rarely had metastases. Therefore, for patients with unfavourable histological features, such as stromal overgrowth, it seems that a close follow up including chest CT and bone scintigram is necessary. Another study showed that phyllodes tumours with an osteosarcomatous component were potentially aggressive neoplasms, with distant metastasis and tumour related death occurring in $38 \%$ and $33 \%$ of patients, respectively. ${ }^{1}$ Moreover, the histological subtype of osteosarcoma in a phyllodes tumour was related to prognosis; the prognosis of osteoclastic and osteoblastic types was poorer than that of the fibroblastic type. ${ }^{1}$ In our present case, the lung metastases were diagnosed as osteoblastic type. Therefore, careful follow up is necessary because the histological findings of both the primary and metastatic lesions include some factors associated with an unfavourable prognosis.

\section{Authors' affiliations}

H Tsubochi, N Sato, T Imai, Department of Thoracic Surgery, Aomori Prefectural Central Hospital, Higashi-Tsukurimichi, Aomori-City, Aomori, 030-8553, Japan

M Kaimori, Department of Pathology, Aomori Prefectural Central Hospital 
Correspondence to: Dr H Tsubochi, Department of Thoracic Surgery, Aomori Prefectural Central Hospital, Higashi-Tsukurimichi, Aomori-City, Aomori, 030-8553, Japan; h-tsubochi@h3.dion.ne.jp

Accepted for publication 11 November 2003

\section{REFERENCES}

1 Silver SA, Tavassoli FA. Osteosarcomatous differentiation in phyllodes tumors. Am J Surg Pathol 1999;23:815-21.

2 Hawkins RE, Schofield JB, Fisher C, et al. The clinical and histologic criteria that predict metastases from cystosarcoma phyllodes. Cancer 1992;69:141-7.

3 Millar EKA, Beretov J, Marr P, et al. Malignant phyllodes tumors of the breast display increased stromal p53 protein expression. Histopathology 1999;34:491-6.
4 Moffat CJ, Pinder SE, Dixon AR, et al. Phyllodes tumours of the breast: a clinicopathological review of thirty-two cases. Histopathology 1995;27:205-18.

5 Cohn-Cedermark G, Rutqvist LE, Rosendahl I, et al. Prognostic factors in cystosarcoma phyllodes. A clinicopathologic study of 77 patients. Cancer 1991;68:2017-22.

6 Graadt van Roggen JF, Zonderland HM, Welvaart K, et al. Local recurrence of a phyllodes tumor of the breast presenting with widespread differentiation to a teleangiectatic osteosarcoma. J Clin Pathol 1998;51:706-8.

7 Chapman AD, Pritchard SC, Yap WW, et al. Primary pulmonary osteosarcoma: case report and molecular analysis. Cancer 2001;91:779-84.

8 Reinfuss M, Mitus J, Duda K, et al. The treatment and prognosis of patients with phyllodes tumor of the breast: an analysis of 170 cases. Cancer 1996;77:910-16.

\section{$\mathrm{ECHO}$}

\section{Nitric oxide synthase 1 and prognosis in cystic fibrosis}

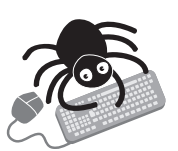

Please visit the Journal of Clinical Pathology website [www. jclinpath.com] for a link to the full text of this article.
A mong patients with cystic fibrosis (CF) the degree of pancreatic involvement is related to the particular mutation of the cystic fibrosis transmembrane conductance regulator gene $(C F T R)$ involved. The severity of lung, liver, or gut disease, however, is much less determined by CFTR genotype. It seems probable, therefore, that other, 'modifier' genes may be involved in determining prognosis. Patients with CF have low concentrations of nitric oxide (NO) in expired air and decreased expression of the nitric oxide synthase 1 gene (NOS1) in upper airway epithelial cells. The number of dinucleotide GT repeats in the $5^{\prime}$-untranslated region (5-UTR) of this gene affects gene expression. Now researchers in Paris have shown that progression of lung disease may be greater in patients whose genes have fewer GT repeats in this region.

They studied 59 adults with CF and 59 healthy controls. On the basis of the number of GT repeats in the 5-UTR region of NOS1 they identified 19 different alleles, the number of repeats ranging from 18 to 36 . The proportion of subjects with more than 27 repeats in 0,1 , or 2 alleles was $24 \%, 54 \%$, and $22 \%$ among controls and $27 \%, 41 \%$, and $32 \%$ among CF patients (no significant difference between patients and controls). A greater number of alleles with more than 27 repeats was associated with an increase in concentration of NO in expired air. Among patients with CF the annual percentage decrease in $\mathrm{FEV}_{1}$ in patients with 0,1 , or 2 alleles with more than 27 repeats was $3.3 \%, 3.2 \%$, and $0.8 \%$. The findings were independent of age, chronic airway colonisation with $P$ aeruginosa or $B$ cepacia, or CFTR genotype.

NOSI genotype may modify the extent of lung damage in patients with cystic fibrosis.

A Thorax 2004;59:156-158. 